\title{
Design of a miniature anamorphic lens with a freeform front group and an aspheric rear group
}

\author{
Chen Xu $\odot{ }^{a}$ Weitao Song $\odot,{ }^{b, *}$ and Yongtian Wang ${ }^{b, c}$ \\ ${ }^{a}$ Hangzhou Dianzi University, School of Automation, Hangzhou, Zhejiang, China \\ ${ }^{\mathrm{b}}$ Beijing Institute of Technology, Beijing Engineering Research Center of Mixed Reality and \\ Advanced Display, School of Optics and Photonics, Beijing, China \\ ${ }^{c}$ AICFVE of Beijing Film Academy, Beijing, China
}

\begin{abstract}
We propose a miniature anamorphic lens design that records wide-screen videos on an ordinary CMOS format. The front group consists of two freeform lenses, which achieve different focal lengths in the two orthogonal directions and thus enable the anamorphic characteristics. The rear group is made of rotationally symmetric aspheric elements that relay the image on the sensor. The annularly stitched extreme-point-based polynomial surface description is proposed to control the extreme point position and the air clearance between elements in the optimization process. An optimization method based on surface upgrade and conversion is adopted in the design. The design result offers an anamorphic ratio of 1.33 and an $f$-number $(f / \#)$ of $\sim 2$, with a field of view of $65.3 \mathrm{deg} \times 35 \mathrm{deg}$. The overall length of the lens is $9.5 \mathrm{~mm}$, which shows an advantage for integration into pocket devices. (C) The Authors. Published by SPIE under a Creative Commons Attribution 4.0 Unported License. Distribution or reproduction of this work in whole or in part requires full attribution of the original publication, including its DOI. [DOI: 10.1117/1.OE.60.6.065104]
\end{abstract}

Keywords: optical design; freeform optics; aspherics.

Paper 20210284 received Mar. 16, 2021; accepted for publication May 25, 2021; published online Jun. 17, 2021.

\section{Introduction}

Anamorphic lenses, which have different magnifications in the $X$ and $Y$ directions, are widely used in cinematography to optically record a wide-screen picture on a regular sensor. For example, such a lens with an anamorphic ratio of 1.33 is able to image a 2:1 format scene on a 3:2 format sensor without loss of effective pixels. Apart from the advantage of full pixel usage to obtain a wider scene, the anamorphic lens shows some unique optical properties favored by the users, such as the artistic horizontal flare caused by bright objects in a scene or the elliptically shaped out-of-focus bokeh. Although first invented for film cinematography, this type of lens design has experienced an expansion from the beginning of this century ${ }^{1-7}$ Lens manufacturers, such as Zeiss AG, Konica Minolta, and Canon Corp., published relevant patents, ${ }^{1-4}$ and some of them have been made into professional movie lenses.

Anamorphic characteristics can be achieved in several ways. In some applications, anamorphic characteristics are obtained from the off-axis effect by inserting prisms or off-axis reflective mirrors. ${ }^{8-10}$ But the most common way to obtain an anamorphic ratio in lens systems is to use optical elements with different optical powers in the $X$ and $Y$ directions, e.g., inserting cylindrical lenses or toroidal lenses into imaging systems. Many efforts have been devoted to exploring the structures of anamorphic lenses of this kind, resulting in three basic types: the front anamorphic attachment type, the middle anamorphic attachment type, and the rear anamorphic attachment type. ${ }^{1-7,11-16}$ Among the three types, the front anamorphic attachment type brings the least aberration and is the conventionally preferred type in fixed-focal systems. ${ }^{11,12}$ Yuan and Sasián ${ }^{12,13}$ studied aberration properties for anamorphic lenses using cylindrical or toroidal elements and proposed a set of aberrations formulas, $\left\{D_{i}\right\}$, for the design process. Zhang et al. ${ }^{14}$ studied the thin lens aberration properties for anamorphic lenses and put forward a paraxial lens design method, and they also studied the condition when the anamorphic ratio is related to object

*Address all correspondence to Weitao Song, swt@bit.edu.cn 
distance and derived the formula to obtain a constant ratio in thin lens approximation. ${ }^{15}$ Different structures of anamorphic lenses using double curvature elements were widely analyzed and discussed by Dodoc ${ }^{16}$ in 2019. Most recently, a big data technique was utilized to explore the feasible solution space for anamorphic lenses. Lippman et al. ${ }^{17}$ generated 1 billion lens models using Monte Carlo searching and studied the first-order solutions for anamorphic zoom lenses. Though the above contributions provide us with powerful tools for the design and analysis of anamorphic lenses, they mainly focus on conventional photographic lenses composed of mostly spherical elements with very few cylindrical, toroid, or aspheric ones. And because of the extra aberrations introduced by double curvature elements and the constraints brought with the anamorphic ratio, such anamorphic photographic lenses have complicated structures and a long total thickness, which makes them unsuitable for integration into pocket devices.

With the development of lens fabrication technologies, aspherical and freeform elements are now widely used in various kinds of lens systems to reduce the number of elements and obtain a more compact form. Various mathematical expressions for aspheric optical surfaces have been published: Zernike polynomials, $Q$-type polynomials, and annularly stitched aspheres. ${ }^{18-20}$ Each surface expression offers unique benefits in the design process and is thus more favorable in certain kinds of designs. Zernike polynomials a have close relationship with aberration coefficients, which helps to design or analyze optical systems. ${ }^{18} Q$-type polynomials are derived from the surface departure slope from the best-fit sphere, and designers can evaluate the surface tolerance or manufacturability based on the coefficients. ${ }^{19}$ Aspheres can be annularly stitched to provide more flexibility in optimization, and their ability is shown in ultra-thin annularly folded systems and disk reading objectives. ${ }^{20}$ When a non-symmetrical surface shape is needed, freeform optics has shown terrific ability for aberration correction in compact off-axis systems ${ }^{21-26}$ and is reported to achieve special optical properties when adopted in coaxial systems. ${ }^{27,28}$ Due to the non-rotationally symmetrical surface shape, freeform elements can serve as the double curvature elements to achieve different focal lengths in the $X$ and $Y$ directions in an anamorphic lens and provide an extra degree of freedom to balance aberrations as compared with cylindrical or toroid ones.

In this paper, we propose a design of a miniature anamorphic lens with a total length within $10 \mathrm{~mm}$. Two freeform elements are adopted to realize the anamorphic characteristics, and a 5-element aspheric rear group transfers the image onto the sensor. In Sec. 2, we describe the overall system specifications and design strategy of our design. To effectively manipulate the shape of wild aspheric elements in the rear group, a special surface expression is derived and utilized. Section 3 shows the performance of the design result. The trade-off between distortion and imaging quality for the design result is discussed, and design results with different surface types are compared in Sec. 4. Finally, the conclusions are drawn in Sec. 5.

\section{Design Strategy}

In this paper, we aim to design a miniature anamorphic lens with the help of freeform and aspheric elements. The specifications for the lens are listed in Table 1. The lens to be designed has a standard focal length that is equivalent to $50 \mathrm{~mm}$ of a full frame camera, but its actual focal length is much smaller by adopting a 1/3" CMOS sensor. A typical 1/3" CMOS sensor has a 4:3 format with the working area of $4.8 \mathrm{~mm} \times 3.6 \mathrm{~mm}$, while in our design, a 3:2 format area on the CMOS is used so that a 2:1 wide-screen effect can be obtained with the 1.33 anamorphic ratio.

Figure 1 illustrates the thin lens approximation for the system. The front group consists of two bicurvature (i.e., freeform) elements, $\mathrm{L}_{1}$ and $\mathrm{L}_{2}$, while the rear group, $L_{r}$, is identical in the $X$ and $Y$ directions. For the afocal front group, it provides a unit magnification in the $X$ direction and $1 / 1.33$ magnification in the $Y$ direction. Thus the rear group can take the rotationally symmetric form.

One reason for choosing the front anamorphic attachment type for our design is its relatively low aberration. Another major reason is that the number and position of non-rotationally symmetrical elements influence the assembling difficulty of the anamorphic lenses in mass production. The azimuth error of freeform elements will bring an extra aberration such as astigmatism. And the assembly difficulty will grow exponentially with the increase of the number of freeform 
$\mathrm{Xu}$, Song, and Wang: Design of a miniature anamorphic lens with a freeform front group...

Table 1 System specifications.

\begin{tabular}{lc}
\hline \hline Focal length in $Y$ direction $(\mathrm{mm})$ & 3.8 \\
Anamorphic ratio $(X / Y)$ & 1.33 \\
Sensor size $\left(\mathrm{mm}^{2}\right)$ & $4.8 \times 3.2$ \\
Full FOV in $Y$ direction $(\mathrm{deg})$ & 64 \\
Full FOV in $X$ direction $(\mathrm{deg})$ & 36 \\
$f / \#$ & $\sim 2$ \\
Total thickness $(\mathrm{mm})$ & $\leq 10 \mathrm{~mm}$ \\
Distortion & $\leq 5 \%$ \\
\hline \hline
\end{tabular}

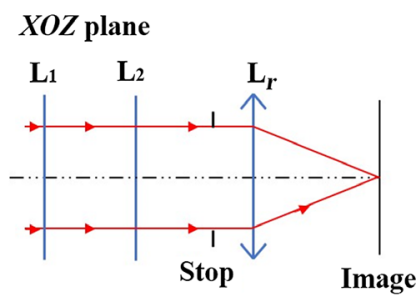

(a)

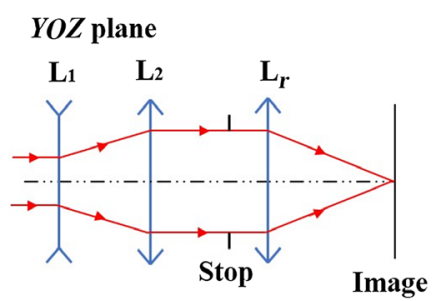

(b)

Fig. 1 The thin lens approximation for the anamorphic lens system.

elements. Thus, only two freeform elements will be used. These two freeform elements are chosen as the front two elements, with the whole lens being divided into two parts, namely a front part that needs to be carefully aligned in azimuth and a rotationally symmetric rear part. This may show advantages over the middle anamorphic type or the rear anamorphic type.

The design process is based on a progressive method, as shown in Fig. 2. The specifications for two sub-systems are obtained, and the initial systems for the front group and the rear group are established, respectively. A combination of the two sub-systems provides the starting point for optimization. The optimization stage includes a coarse optimization phase to explore

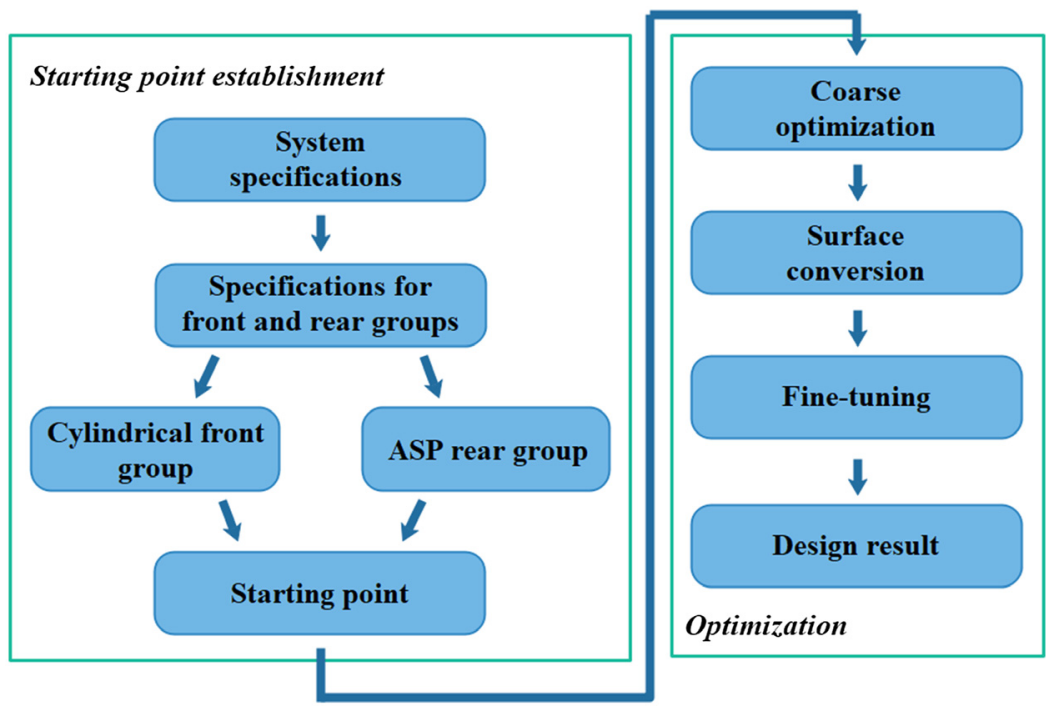

Fig. 2 The flowchart of the design process. 
different structures and a fine-tuning phase that utilizes upgraded surface expressions and all possible variables to find the best result.

The material for each element is chosen following the basic idea that positive lens elements have a low refractive index and high Abbe number while negative lenses have a high refractive index and low Abbe number. Thus, we chose EP-type material (Mitsubishi Gas Chemical $n d=1.620, V d=23.5$ ) for negative lenses and APEL-type material (Mitsui Chemicals, $n d=1.535, V d=56.1$ ) for positive lenses for the starting point.

\subsection{Starting Point for the Anamorphic Front Group}

The anamorphic group takes the two-element form and is first designed to be an afocal inverse telescope in the $Y$ direction, and all four surfaces have an infinity radius in the $X$ direction. This can be achieved by a negative cylindrical lens followed by a positive one. The anamorphic ratio of the front group is expressed by $-f_{y 2} / f_{y 1}$, where $f_{y 1}$ and $f_{y 2}$ are focal lengths of the first and the second cylindrical lens, respectively. Thus, the starting point of the front group is modeled in the lens design software with two cylindrical lenses, and the stop is placed $0.5 \mathrm{~mm}$ behind the second lens. A lens model with a 4.93- $\mathrm{mm}$ focal length is placed after the stop surface as an equivalent of the rear group. An optimization is performed to obtain the optimized surface curvatures while maintaining the target focal length in the $Y$ direction. The result is shown in Fig. 3(a).

\subsection{Starting Point for the Rear Group}

The rotationally symmetric rear group transfers the image plane in the $X$ and $Y$ directions onto the sensor. To balance the size of each element, the stop surface is placed on the front surface of the first lens in the rear group. A 5-element lens configuration is chosen as the starting point. The field angle is set in accordance with the output ray angles of the front anamorphic group.

Considering the system parameters of the rear group, the 5-element, "positive-negativepositive-positive-negative" structure is adopted for the starting point. For such a miniature lens, it is usually impossible to obtain a starting point with an acceptable imaging performance with sphere elements. Aspheres are needed in the starting point, and wild aspheres that resemble seagull wings usually exist at the starting point. We derived a set of polynomials to describe such wild aspheres with the following concerns. First, it should use very few coefficients to express the seagull surface so that designers can easily establish such surfaces at the starting point. Second, the position of the extreme point can be easily manipulated by the designer, enabling an interactive way to adjust the surface shape.

To facilitate the adjustment of the extreme point, its position is incorporated into the surface description as parameters. An annually stitched expression is adopted in the way that the area

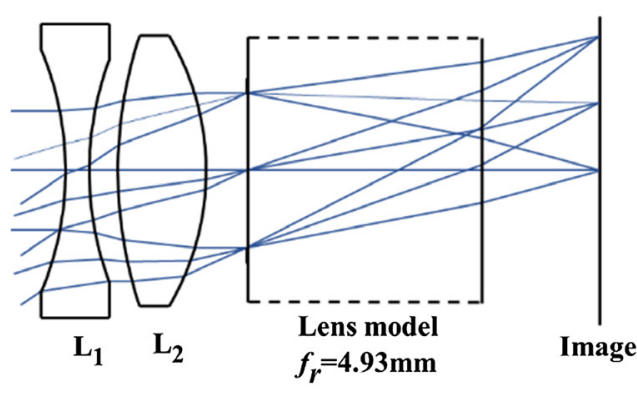

$2.00 \mathrm{~mm}$

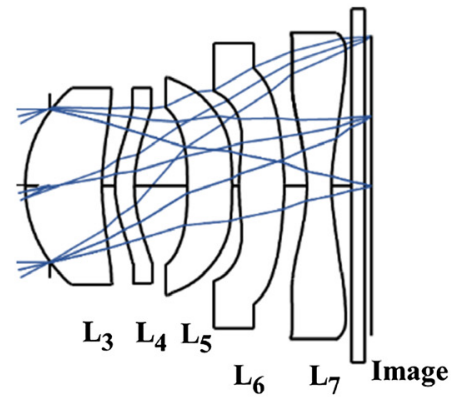

(b)

(a)

$\underline{2.00 \mathrm{~mm}}$

Fig. 3 The starting points for the front group and the rear group. (a) The anamorphic front group consisting of two cylindrical lenses. (b) The rotationally symmetric rear group consisting of five aspheric elements. 
$\mathrm{Xu}$, Song, and Wang: Design of a miniature anamorphic lens with a freeform front group...

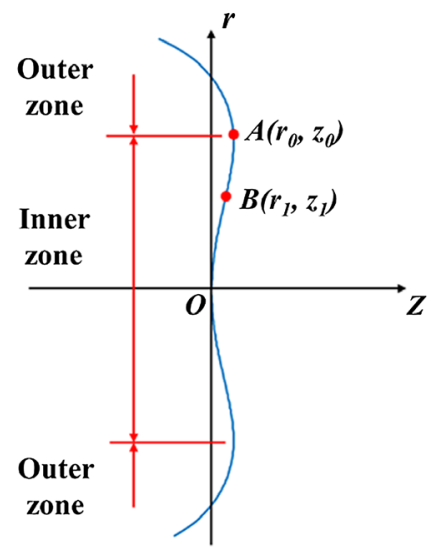

Fig. 4 Coordinate representation for the ASEP surface. $A\left(r_{0}, z_{0}\right)$ is the extreme point, and $B\left(r_{1}, z_{1}\right)$ is the point where $Z^{\prime \prime}(r)=0$.

from the axial point to the extreme point is described with one set of polynomials and the area beyond the extreme point with another set of polynomials.

Suppose the surface profile is expressed by $z(r)=f_{b}(r)+\sum_{i} a_{i} P_{i}(r)$, and as illustrated in Fig. 4, the coordinate $r_{0}$ of the extreme point $A$ and the coordinate $r_{1}$ of point $B$ are incorporated into the surface expression. We have one possible constraint set for the $f_{b}$ and $P_{i}$ in Eq. (1),

$$
\left\{\begin{array} { l } 
{ f _ { b } ( 0 ) = 0 } \\
{ f _ { b } { } ^ { \prime } ( 0 ) = 0 } \\
{ f _ { b } ( r _ { 0 } ) = z _ { b } } \\
{ f _ { b } { } ^ { \prime } ( r _ { 0 } ) = 0 } \\
{ f _ { b } ^ { \prime \prime } ( r _ { 1 } ) = 0 }
\end{array} \quad \text { and } \quad \left\{\begin{array}{l}
P_{i}(0)=0 \\
P_{i}^{\prime}(0)=0 \\
P_{i}^{\prime}\left(r_{0}\right)=0 \\
P_{i}^{\prime \prime}\left(r_{1}\right)=0
\end{array}\right.\right.
$$

where $z_{b}$ is a coefficient that adjusts the shape. For the optical area in the outer zone, another set of $b_{i}$ is used for $P_{i}$, and the continuity is self-guaranteed. The surface expression for the annularly stitched extreme-point-based polynomial (ASEP) is shown in Eq. (2),

$$
\begin{aligned}
z(r)= & -\frac{3 z_{0}\left(r_{0}-2 r_{1}\right)}{r_{0}^{3}\left(r_{0}^{2}-6 r_{0} r_{1}+6 r_{1}^{2}\right)} r^{4}+\frac{4 z_{0}\left(r_{0}^{2}-3 r_{1}^{2}\right)}{r_{0}^{3}\left(r_{0}^{2}-6 r_{0} r_{1}+6 r_{1}^{2}\right)} r^{3}-\frac{6 z_{0} r_{1}\left(2 r_{0}-3 r_{1}\right)}{r_{0}^{2}\left(r_{0}^{2}-6 r_{0} r_{1}+6 r_{1}^{2}\right)} r^{2} \\
& + \begin{cases}\sum_{i} a_{i}\left(r^{2 i+4}-\frac{i+2}{i+1} r_{0}^{2} r^{2 i}\right) & 0 \leq r \leq r 0 \\
\sum_{i} b_{i}\left(r^{2 i+4}-\frac{i+2}{i+1} r_{0}^{2} r^{2 i}\right) & r>r 0\end{cases}
\end{aligned}
$$

The proposed surface description is implemented as the user-defined surface type in the lens design software CODE $\mathrm{V}^{29}$ with $r_{0}, r_{1}, z_{b}, a_{i}$, and $b_{i}$ used as variables in optimization.

The optical surfaces in the rear group are first modeled as a conic surface, and then low-order aspheric terms are added. ASEP works as the tool for the design to tune the shape of wild aspheres. During optimization, once the optical surface turns into the seagull shape, it is converted to the ASEP expression. Manual adjustment of the extreme points' positions on the ASEP surfaces is performed when the optimization process stagnates. The shape of the outer zone on ASEP is constantly modified to address some ray-tracing failure problems.

The starting point for the rear group is obtained after the optimization process, which is shown Fig. 3(b). The front surface of $\mathrm{L}_{6}$ and both surfaces of $\mathrm{L}_{7}$ are described by ASEP, and the remaining surfaces are aspheric. During this process, the focal length is $4.93 \mathrm{~mm}$, the abovementioned two materials are used, and a local optimization is performed to achieve a relatively good imaging quality. 


\subsection{Constraints}

When performing first-order parameter control, it is important to obtain the real paraxial focal length of the system. In particular, for $X Y$-polynomial (XYP) and ASEP surfaces, the coefficients for $x^{2}, y^{2}$, or $r^{2}$ will contribute to the focal length. Thus, the default effective focal length evaluated by the lens design software may not be accurate enough. For optimizing the XYP surface obtained by surface fitting from the anamorphic asphere surface (AAS), the $x^{2}$ term is used to control the anamorphic ratio; otherwise, the widened field of view (FOV) may be obtained mainly by the negative distortion in the $Y$ direction. The paraxial curvatures are obtained using Eq. (3),

$$
\left\{\begin{array}{l}
C U X=\frac{d^{2} z / d x^{2}}{\left[1+(d z / d x)^{2}\right]^{3 / 2}}=C U X_{B}+2 a * \text { sign_flag } \\
C U Y=\frac{d^{2} z / d y^{2}}{\left[1+(d z / d y)^{2}\right]^{3 / 2}}=C U Y_{B}
\end{array},\right.
$$

where $C U X$ and $C U Y$ denote the real paraxial curvatures in the $X$ and $Y$ directions, respectively, $C U X_{B}$ and $C U Y_{B}$ denote the curvatures provided by the conic base, $a$ denotes the coefficient for the $x^{2}$ term, and sign_flag is either 1 or -1 according to whether $a$ adds to the absolute curvature.

When wild aspheres exist in the system, the physical interference cannot be fulfilled effectively by center thickness or edge thickness. For this condition, the axial air clearance at the extreme point is controlled to avoid lens overlapping. Other physical constraints are set to benefit the fabrication of each element using the precision molding method. For example, the minimum center thickness or edge thickness of each element is set to be no thinner than $0.25 \mathrm{~mm}$. The edge thickness in the air between each element is controlled to be greater than $0.1 \mathrm{~mm}$. The total thickness is controlled to be within $10 \mathrm{~mm}$.

For such a non-rotationally symmetric system, the default distortion control code provided by the lens design software cannot meet the demand. In particular, when XYP surfaces are used, the distortion in the $X$ direction is not evaluated. In our design process, the real image height is used as the definition of the field angle, which ensures that the entire rectangular image area is evaluated even though distortion exists. In this case, the incident angle of the principle rays of the marginal fields is restricted according to the $f \cdot \tan \theta$ model to limit the distortion in the $X$ and $Y$ directions.

\subsection{Optimization}

A progressive optimization strategy with surface upgrade is adopted. At the beginning of the coarse optimization phase, the cylindrical front group and the aspheric rear group are combined, and a preliminary optimization is performed to make all rays have the right path and no raytracing errors exist while maintaining the focal length. Then, the cylindrical surfaces are converted into Y-toroid (YTO) or AAS surfaces. The curvatures in the $X$ and $Y$ directions as well as aspheric coefficients are set as variables in optimization. The front group no longer needs to be afocal. Stricter constraints on anamorphic ratio and distortion are added. Global optimizations are repeatedly performed to obtain results with the best MTF performance.

In the fine-tuning phase, the YTO or AAS surfaces are upgraded to $X Y$-polynomials (XYP) freeform surfaces that are able to provide more degrees of freedom in optimization. The ASEP surfaces are converted back to aspheres for fabrication purposes. This conversion process cannot be realized by mathematical transformation; thus, a surface fitting method is used. When all four surfaces of the front group are XYP surfaces, the fine-tuning optimization is performed. The MTF values between different field angles are balanced, and the distortion is carefully adjusted in this stage.

\section{Design Result and Analysis}

A design result is obtained using the method described in Sec. 2; its code is in Ref. 30. Figures 5(a) and 5(b) show the 2D layout of the design result in the $X O Z$ and $Y O Z$ planes, respectively. The front and rear surfaces of $\mathrm{L}_{1}$ and $\mathrm{L}_{2}$ are all freeform surfaces described by 
$\mathrm{Xu}$, Song, and Wang: Design of a miniature anamorphic lens with a freeform front group...

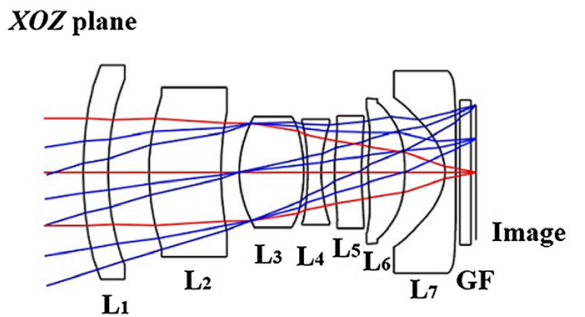

L1

\section{YOZ plane}

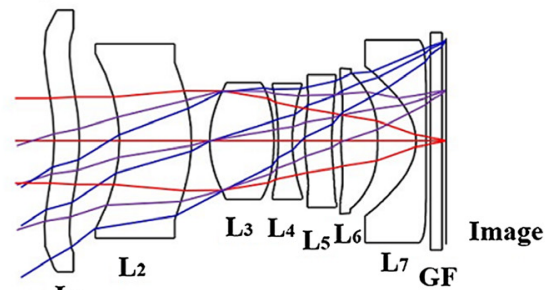

$2.00 \mathrm{~mm}$

(a) (b)

Fig. 5 The 2D layout of the design result. The layout in the $X O Z$ plane is shown in (a) and the layout in the $Y O Z$ plane is shown in (b).

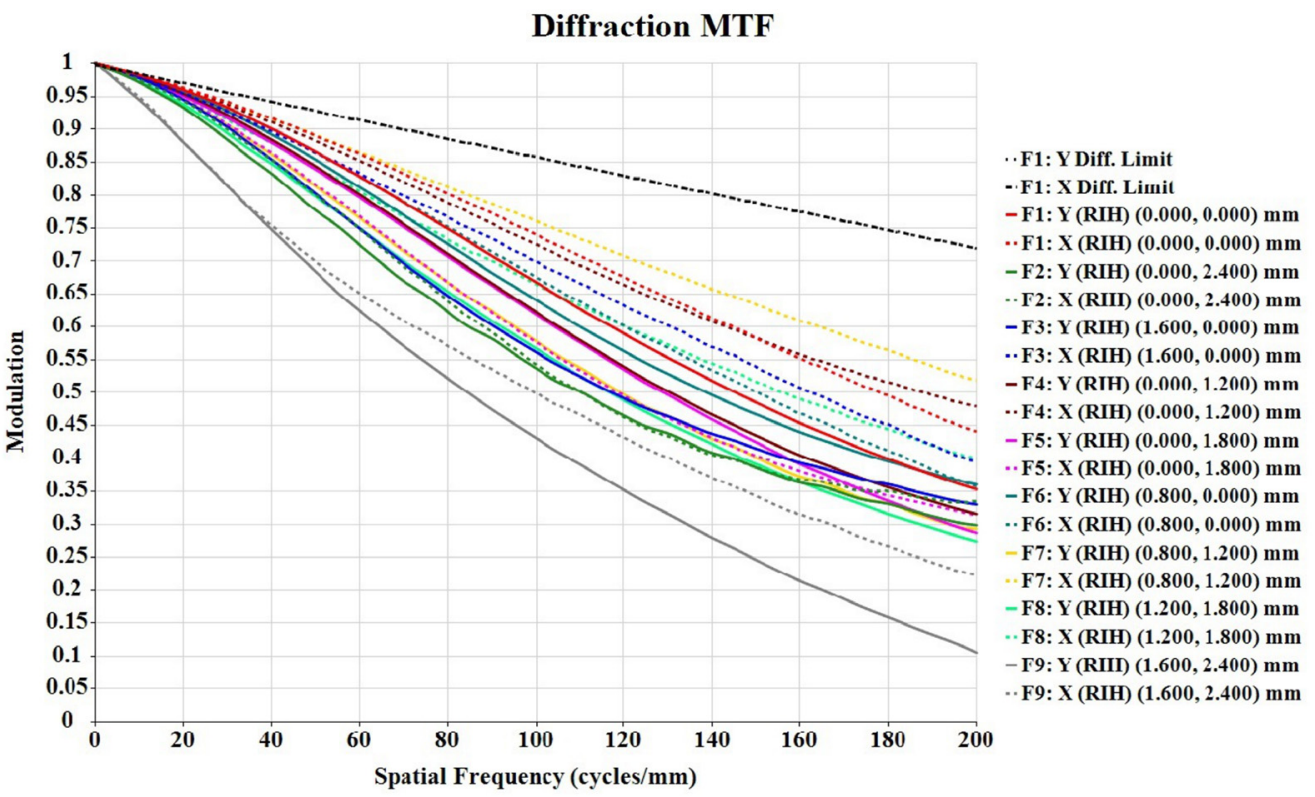

(a)

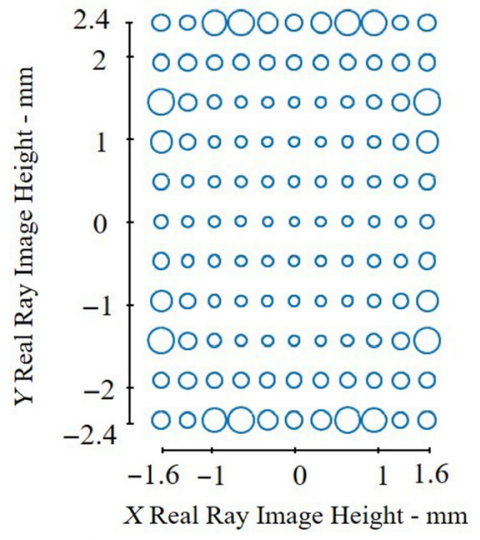

Maximum $=0.0087369$

Minimum $=0.003152$

Average $=0.0049904$

Std Dev $=0.0014904$

(b)
Distortion Grid

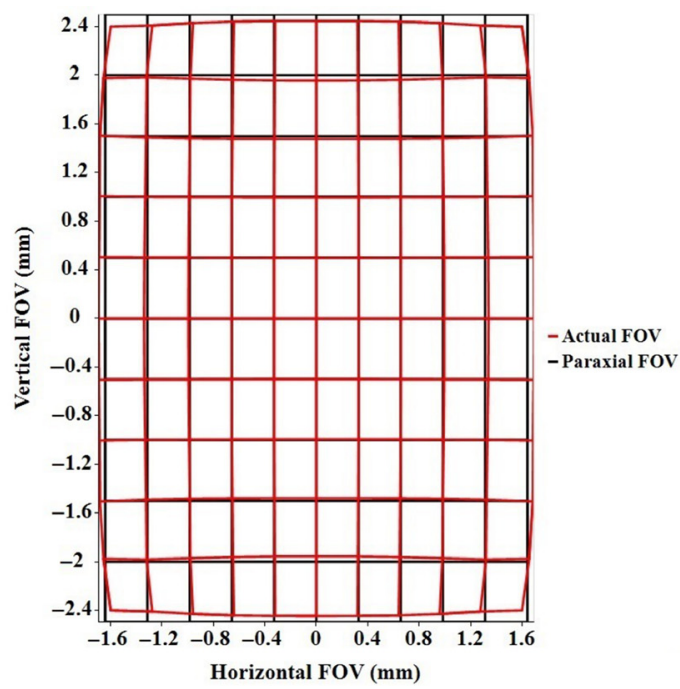

(c)

Fig. 6 The performance of the design result. (a) MTF curves, (b) the RMS spot diameters over the field, and (c) the distortion grid. 


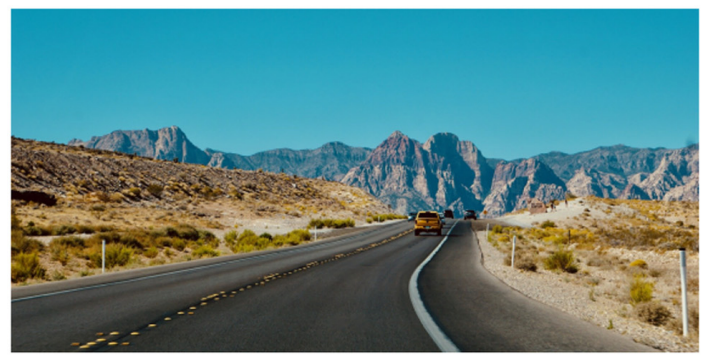

(a)

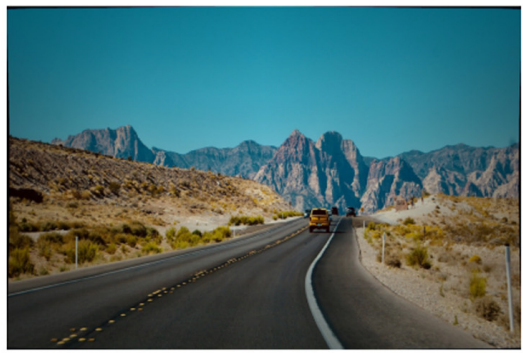

(b)

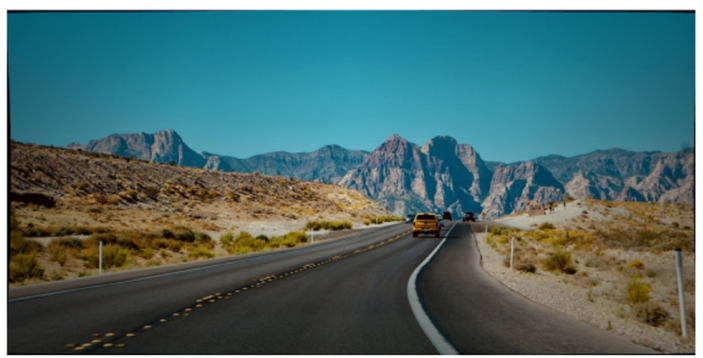

(c)

Fig. 7 Image simulation of the design result. (a) The original scene, (b) the simulated image captured by the design result, and (c) the restored image for wide screen display.

XYP, and the remaining elements are aspheric. The overall length of the design is $9.5 \mathrm{~mm}$, which makes it easy to be integrated into mobile devices. The $f / \#$ in the $X$ and $Y$ directions are 1.97 and 1.9 , respectively.

The MTF curves and the field map of the design result are shown in Fig. 6. The average MTF value of all field angles reaches 0.35 at $200 \mathrm{lp} / \mathrm{mm}$. The field map shows the RMS spot diameters over the whole FOV, which implies a balanced performance over fields. Except for the peripherical area, the RMS spot size is within $5 \mu \mathrm{m}$. The maximum tangential distortion of the design result is about $3.55 \%$, which is negligible for video watching and can be further calibrated through post-processing.

The anamorphic characteristics of the design result are illustrated by the image simulation in Fig. 7. Figure 7(a) shows the original 2:1 format scene, and Fig. 7(b) shows the 3:2 format simulated image captured by the design result using CODE V. To obtain the wide-screen effect, the simulated image is restored by stretching to the $2: 1$ format, and the final image is shown in Fig. 7(c). In the simulation, the residual distortion causes a slim, black area at both ends in the horizontal direction. But for real use, these areas correspond to a slightly larger horizontal FOV.

\section{Discussion}

\subsection{Imaging Quality and the Distortion}

In the design of a miniature anamorphic lens, a trade-off between the anamorphic ratio and the distortion shows up. A system with a smaller anamorphic ratio and greater negative distortion in the $Y$ direction may provide the same FOV in the $X$ and $Y$ directions as a system with a greater 
anamorphic ratio and negligible distortion. And in our design process, the latter often shows better performance in image quality, i.e., higher MTF curves. Figure 8 shows one design result with an anamorphic ratio of 1.33 , and its maximum distortion reaches $11 \%$ in the tangential direction. Due to the large negative distortion in the $Y$ direction, the paraxial focal length is $4.13 \mathrm{~mm}$ in the $Y$ direction while maintaining an FOV of $64 \mathrm{deg}$ in the $Y$ direction. The FOV in the $X$ direction decreases to $32 \mathrm{deg}$. The ratio of FOVs in the $Y$ and $X$ directions is equivalent to a distortion-free anamorphic system with an anamorphic ratio of 1.45. Hence, under some circumstance in which image quality is more important than distortion performance, the negative distortion strategy can be adopted.

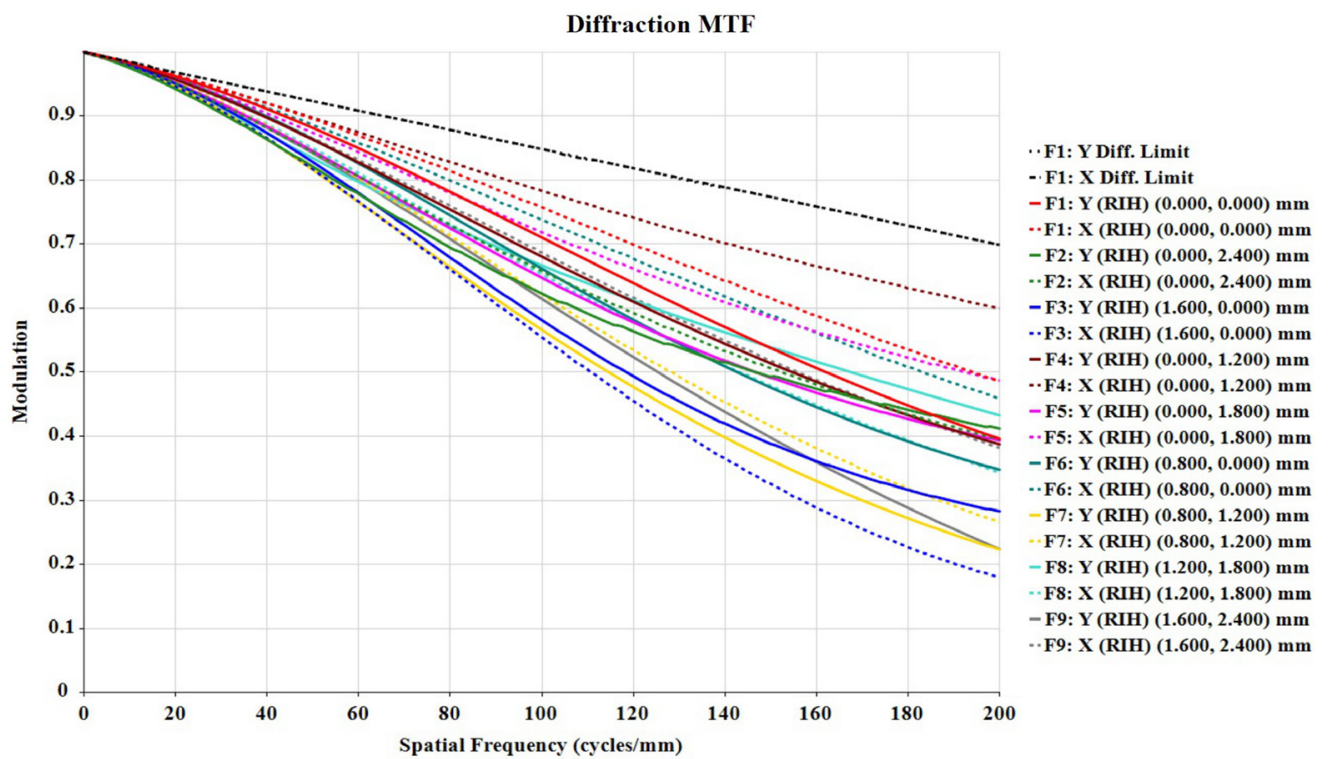

(a)

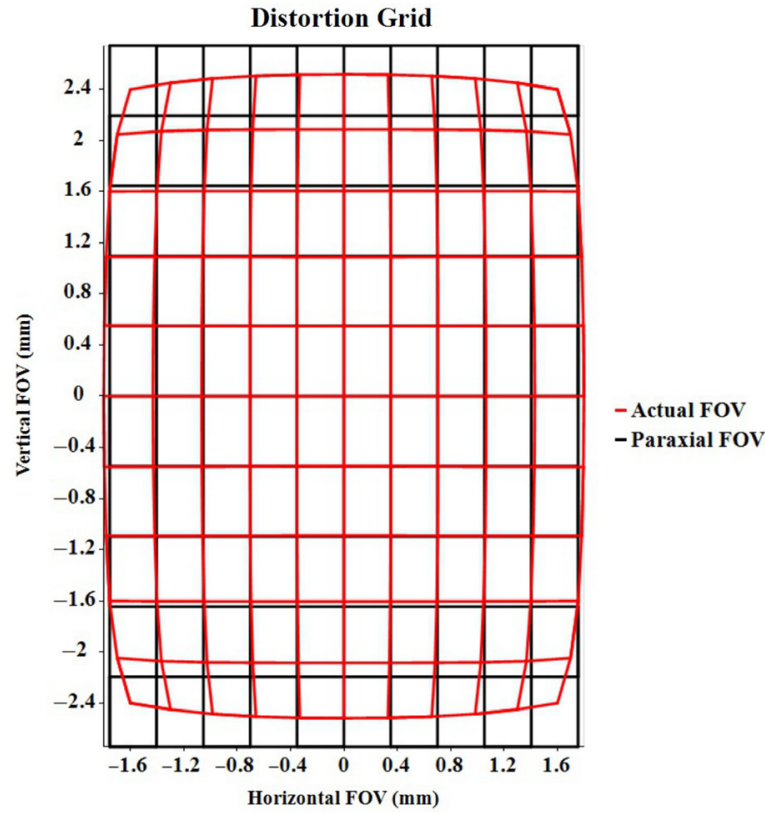

(b)

Fig. 8 Performance of another design result with higher MTF curves but larger distortion. (a) The MTF curves of the design result. (b) The distortion grid. The translucent pink area indicates the active area of the sensor. It can be inferred from (b) that the anamorphic effect is increased by the large negative distortion in the $Y$ direction. 
$\mathrm{Xu}$, Song, and Wang: Design of a miniature anamorphic lens with a freeform front group...

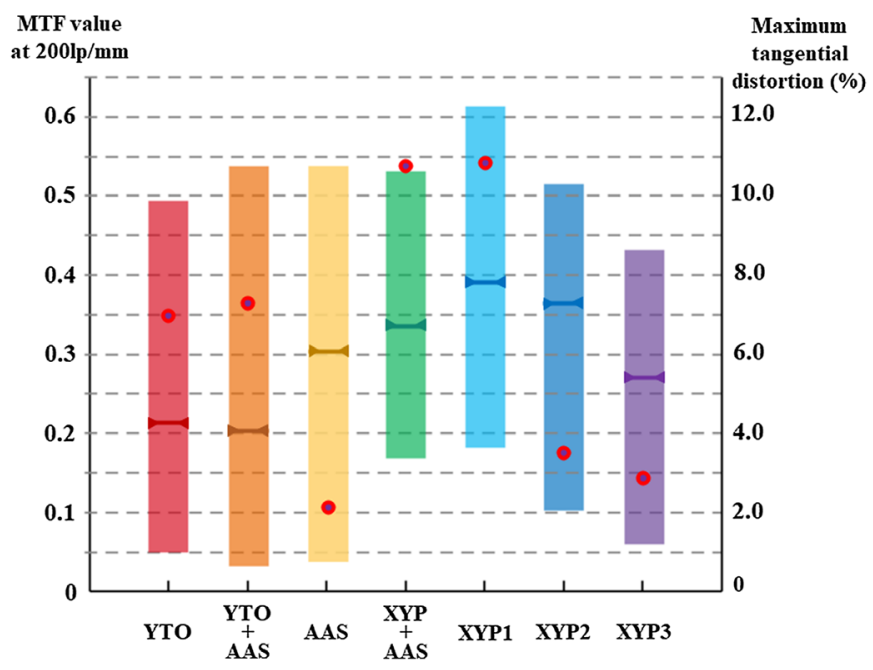

Fig. 9 The MTF values at $200 \mathrm{lp} / \mathrm{mm}$ and the maximum distortion performance for design results with different surface types. "YTO" denotes the design with two Y-toroid elements; "YTO+AAS" denotes the design with one $Y$-toroid element and one AAS element; "AAS" denotes the design with two AAS elements; "XYP+AAS" denotes the design with one AAS element and one XYP element; "XYP1," "XYP2," and "XYP3" are three designs with 2 XYP elements each. Colored bars stand for the range of MTF values at $200 \mathrm{lp} / \mathrm{mm}$ over the entire FOV, and the line in the bar illustrates the average MTF value. Red circles denote the maximum tangential distortion (\%) for each design result. All design results are optimized with similar constraints except that the distortion of the "AAS" and "XYP3" designs is carefully controlled.

Three designs with the XYP front group but with different emphases on distortion are shown in Fig. 9, among which the "XYP2" is the design result in Sec. 3. This further shows the "tradeoff" between distortion and imaging quality for a certain structure.

\subsection{Comparison between Front Groups with Different Surface Expressions}

Considering that cylindrical is a special subset of both toroid surfaces and anamorphic aspheres, two upgrade paths can be adopted. Thus, to analyze the influence of surface types in optimization, the results with different surface types are illustrated in Fig. 9. As shown in Fig. 9, adding aspheric or freeform terms to the front group helps to improve the MTF values. Surfaces with more degrees of freedom tend to more flexible surfaces that improve MTF values at the cost of distortion. Thus, distortion control is more important for AAS or XYP designs. The design of the anamorphic front group with two AAS or XYP elements can achieve comparable imaging quality, while XYP offers more degrees of freedom to improve the image quality of the worst sampled field angle.

\section{Conclusions}

In this paper, we have designed a miniature anamorphic lens by employing two freeform lenses as the front group and five aspheric lenses as the rear group. The overall system achieved an anamorphic ratio of 1.33 and an $f / \#$ of 1.9 in the $Y$ direction, with an FOV of $65.3 \mathrm{deg} \times 35 \mathrm{deg}$. The total length of the design result was as short as $9.5 \mathrm{~mm}$. The design method and constraints in optimization were detailed, and the design result was analyzed. This paper also illustrated a condition in which freeform elements were adopted in axial systems to obtain some special optical ability, i.e., the anamorphic characteristics. In the future, we will study the design of a miniature wide-angle anamorphic lens that provides an ultra-wide FOV in the horizontal direction for landscape photography. 


\section{Acknowledgments}

This research was supported by Zhejiang Provincial Natural Science Foundation of China (No. LQ19F050006) and the National Natural Science Foundation of China (No. 62005069). We would also like to thank Synopsys for the education license of CODE V.

\section{References}

1. R. Nurishi and T. Yoshimi, "Anamorphic converter, lens device using the same, and imagetaking device using the same," U.S. Patent 7,113,344B2 (2006).

2. S. Ohzawa, M. Inoue, and M. Imoto, "Ultra wide angle imaging optical system, ultra wide angle imaging lens device, and image sensing apparatus," U.S. Patent 7,457,044B2 (2008).

3. M. Pretorius, "Anamorphic imaging objective," U.S. Patent 8,174,773B2 (2012).

4. A. Dodoc et al., "Anamorphic objective," U.S. Patent 8,858,099B2 (2014).

5. A. V. Navarro, A. V. Navarro, and C. A. Garrido, "Anamorphic lens," U.S. Patent 9,063,321B2 (2015).

6. I. A. Neil, "Anamorphic objective zoom lens," U.S. Patent 9,239,449B2 (2016).

7. J. B. Caldwell, "Anamorphic optical system with relative small squeeze ratio but with relatively pronounced residual anamorphic characteristics," U.S. Patent 10,126,533B1 (2018).

8. Y-J Chen and J.-W. Pan, "Designing an anamorphic illumination system with an RTIR prism for a tilt-and-roll-pixel-type projector," Appl. Opt. 59(12), 3530-3537 (2020).

9. C. Xu et al., "Design of all-reflective dual-channel foveated imaging systems based on freeform optics," Appl. Opt. 55(9), 2353-2362 (2016).

10. K. V. Schenau et al., "Imaging performance of the EUV high NA anamorphic system," Proc. SPIE 9661, 96610S (2015).

11. S. Yuan and J. Sasián, "Aberrations of anamorphic optical systems. I: the first-order foundation and method for deriving the anamorphic primary aberration coefficients," Appl. Opt. 48(13), 2574-2584 (2009).

12. S. Yuan and J. Sasián, "Aberrations of anamorphic optical systems. II: primary aberration theory for cylindrical anamorphic systems," Appl. Opt. 48(15), 2836-2841 (2009).

13. S. Yuan and J. Sasián, "Aberrations of anamorphic optical systems. III: the primary aberration theory for toroidal anamorphic systems," Appl. Opt. 49(35), 6802-6807 (2010).

14. J. Zhang et al., "Thin lens aberrations for anamorphic lenses," Appl. Opt. 58(1), 182-188 (2019).

15. J. Zhang et al., "Paraxial lens design of anamorphic with a fixed anamorphic ratio," OSA Continuит 2(4), 1430-1454 (2019).

16. A. Dodoc, "Anamorphic prime and zoom lenses," Proc. SPIE 11106, 1110603 (2019).

17. D. H. Lippman, D. S. Teverovsky, and J. L. Bently, "Monte Carlo first-order design method for anamorphic cinema zoom lenses," Opt. Eng. 60(5), 051203 (2021).

18. J. Ye et al., "Review of optical freeform surface representation technique and its application," Opt. Eng. 56(11), 110901 (2017).

19. G. W. Forbes, "Shape specification for axially symmetric optical surfaces," Opt. Express 15(8), 5218-5226 (2007).

20. D. Cheng et al., "Optical description and design method with annularly stitched aspheric surface," Appl. Opt. 54(34), 10154-10162 (2015).

21. D. Cheng et al., "Design of an optical see-through head-mounted display with a low f-number and large field of view using a freeform prism," Appl. Opt. 48(14), 2655-2668 (2009).

22. F. Z. Fang et al., "Manufacturing and measurement of freeform optics," CIRP Ann. Manuf. Technol. 62(2), 823-846 (2013).

23. K. Takahashi, "Development of ultrawide-angle compact camera using free-form optics," Opt. Rev. 18(1), 55-59 (2011).

24. T. Yang, G. F. Jin, and J. Zhu, "Automated design of freeform imaging systems," Light Sci. Appl. 6(10), e17081 (2017).

25. L. Wei et al., "Design and fabrication of a compact off-axis see-through head-mounted display using a freeform surface," Opt. Express 26(7), 8550-8565 (2018). 
26. S. He, Y. Meng, and M. Gong, "Freeform lens design to eliminate retroreflection for optical systems," Appl. Opt. 57(5), 1218-1224 (2018).

27. J. Chen et al., "Design and tests of a high-performance long-wave infrared refractive thermal imager: freeform lens in coaxial system," Appl. Sci. 7(11), 1195 (2017).

28. X. Wu et al., "Transverse image translation using an optical freeform single lens," Appl. Opt. 54(28), E55-E62 (2015).

29. CODE V, "Reference manual," Synopsys (2020).

30. https://github.com/ChenXu-2021/AnamorphicLensDesign.

Chen $\mathrm{Xu}$ received his $\mathrm{BS}$ degree in optoelectronics and his $\mathrm{PhD}$ in optical engineering from Beijing Institute of Technology, Beijing, China, in 2012 and 2018, respectively. He is currently with Hangzhou Dianzi University, China. His research interests include optical design, freeform optics, and biomedical optics. He is a member of SPIE.

Weitao Song received his BS degree and PhD from Beijing Institute of Technology in 2010 and 2016, respectively. Currently, he is an associate professor at Beijing Institute of Technology, China. His research interests include human-computer interaction and virtual and augmented reality.

Yongtian Wang received his BS degree in precision instrumentation from Tianjin University, China, in 1982, and his $\mathrm{PhD}$ in optics from the University of Reading, U.K., in 1986. He is currently a professor at the School of Optics and Photonics, Beijing Institute of Technology, China, and he works in AICFVE of the Beijing Film Academy, China. His research interests include optical design and CAD, optical instrumentation, and virtual reality and augmented reality technologies and applications. 